\title{
Tendencias sociopolíticas-económicas en la inclusión del inglés en los planes curriculares normalistas mexicanos
}

\author{
Social-political-economic trends in the inclusion of English \\ in Mexican Curriculum of initial teacher schools
}

Beatriz Cecilia Roldán Virgen ${ }^{*}$

Recibido: 29 de mayo de 2020 Aceptado: 5 de agosto de 2020 Publicado: 31 de enero de 2021

To cite this article: Roldán, B. C. (2021). Tendencias sociopolíticas-económicas en la inclusión del inglés en los planes curriculares normalistas mexicanos. Márgenes, Revista de Educación de la Universidad de Málaga, 2 (1), 41-60

DOI: https://doi.org/10.24310/mgnmar.v2i1.9473

\section{RESUMEN}

Este estudio analiza diez reformas educativas para las escuelas normales de México. Se pretende responder la pregunta sobre cuáles han sido las tendencias político-económicas que han marcado la inclusión o no inclusión de la asignatura de Inglés en los planes curriculares normalistas de 1820 a 2018. Este rango de tiempo se refiere, con el primero, al año en que emergen las primeras escuelas normales y, con el último, al año en que se implementa una reforma normalista reciente. El estudio se realiza bajo un paradigma cualitativo, con enfoque metodológico de análisis documental, al que se le aplica la noción de genealogía de Michel Foucault para abordar los acontecimientos que influyen en que se incluya o no el inglés en los planes curriculares normalistas. En los hallazgos se evidencian ciclos de altibajos con contracciones y expansiones tanto sociales y políticas como educativas que actúan ya sea como fuerzas revitalizadoras o eliminadoras de la asignatura de inglés en el currículo normalista mexicano.

Palabras clave: lenguas extranjeras; reforma educativa; formación inicial de profesores; política educativa

\section{ABSTRACT}

This study analyzes ten educational reforms for initial teacher schools in Mexico. It intends to answer the question: What are the political and economic trends that have marked the inclusion or non-inclusion of the English subject in the curriculum plans for teachers' schools from 1820 to 2018? This time range refers firstly, to the moment when teachers' training schools emerged and to the last, when the most recent reform was implemented. A qualitative paradigm is adopted in order to follow a documentary analysis. Michel Foucault's notion of genealogy is applied to events and social context that surrounds English within curricular plans. The findings recognize cycles of ups and downs with both social, political and educational contractions and expansions that act as either revitalizing or eliminating forces of the English subject in the Mexican curriculum for initial teacher's schools.

Keywords: foreign languages; educational reform; initial teacher education; educational policy 


\section{INTRODUCCIÓN}

El devenir tecnológico y social ha acentuado la comunicación y la conexión intercontinental en las primeras décadas del siglo XXI. Este rasgo ha penetrado en la educación por una relación intrínseca que propicia acceso y difusión en el orden de las ideas, procesos, dinámicas, engranajes y cambios en ambientes educativos. Alcanzar tales despliegues es posible si se comparten códigos, lenguajes y sistemas de significación. Como instrumento de acceso, el idioma inglés ha logrado girar la balanza hacia una posición diferencial en referencia al resto de idiomas. Las últimas dos reformas para la formación inicial de maestros han tomado esta incidencia como primordial en las definiciones de su política educativa ${ }^{1}$ (CDPPE, 2014). Sin embargo, los cambios curriculares normalistas no siempre han involucrado la asignatura de lengua extranjera o al inglés. De aquí surge un interés por indagar sobre la trayectoria del inglés en el currículo de las escuelas normales.

La tradición investigativa ha abordado la historia de la educación normalista mexicana en profundidad con poca atención a la asignatura de Inglés en los planes de estudio (Estrada, 1992; Arnaut, 1996; Ducoing, 2004; Ducoing, 2013; Navarrete, 2015). El documento de Estrada (1992) comprende un recuento de las primeras obras educativas en el México posindependista y concluye que durante dicho periodo se actuó con el afán de proveer educación básica y la necesidad de formar maestros en todo un territorio aún vasto y difícil de articular. En el texto, "Historia de una profesión”, Alberto Arnaut elabora una reconstrucción del normalismo mexicano y en su conclusión descifra diferentes estadios temporales en una perspectiva política e institucional, que abarca de finales del siglo XIX hasta la última década del siglo XX.

La historiografía de las escuelas normales nos remite a las obras de Patricia Ducoing y Zaira Navarrete. En su estudio, denominado “Origen de la Escuela Normal Superior” (Ducoing, 2004), la autora finaliza argumentando que México presenta la ambivalente salida de formar docentes tanto en las escuelas normales, para educación básica, como en las universidades, para media superior y universitaria; a diferencia de algunos países latinoamericanos en los que es la universidad la encargada de la formación de docentes para todo el sistema educativo. Por su parte, Navarrete (2015) reconoce los enfoques teóricos que se han trasegado en las escuelas normales: el socialismo, la escuela activa, la teoría del capital humano, el marxismo y el modelo reflexivo, paralelos a los cambios curriculares ocurridos durante el siglo pasado y parte del actual.

Estos trabajos presentados ponen en relieve la escasa presencia de investigaciones centradas en el tema de los idiomas extranjeros dentro del currículo de las escuelas normales mexicanas; a excepción de Muñoz (2015), quien se centra en describir la presencia del inglés en los programas desde su origen, en 1885, hasta 2009. Sin embargo, el texto de Muñoz carece de análisis y no contempla la entrada nuevamente al poder presidencial del partido hegemónico (Partido Revolucionario Institucional) de 2012 a 2018, que se avocaría a una reforma trascendental en 2017, la cual generaría cambios cruciales en los programas para las escuelas normales. Tampoco recoge la elección de un gobierno de oposición en 2018 (Movimiento de Renovación Nacional) con una visión contrarreformista de lo hecho por su antecesor en materia educativa.

1 El Comité de Dirección de Política y Práctica Educativa en 2014 emite una serie de recomendaciones en cuanto a políticas educativas para el desarrollo de competencias lingüísticas para la escolaridad. 
Lo anteriormente descrito plantea los siguientes cuestionamientos: ¿en cuáles reformas el gobierno en turno ha incorporado la asignatura de Inglés al plan curricular?, ¿cuáles han sido los contextos sociopolítico y económico en torno a la inclusión o no inclusión del inglés en la reforma curricular? Las respuestas a tales interrogantes aportarán un entendimiento al devenir del inglés como asignatura en la escena normalista y a los diversos sucesos que envuelven su presencia o ausencia del currículo de las escuelas normales de México. De ahí que la pregunta que este estudio propone responder gira en torno a cuáles son las tendencias político-económicas que han marcado la inclusión o no inclusión de la asignatura de Inglés en los planes curriculares normalistas desde 1820 a 2018. Esta interrogante conduce a un análisis historiográfico-documental cimentado en las posturas teóricas de Michel Foucault, concernientes al estudio de la genealogía, cuyo marco da cuenta del sentido historiográfico y del análisis de fuerzas implicadas.

\section{POSTURA TEÓRICA PARA EL ANÁLISIS}

Se acude a la genealogía de Michel Foucault como el marco que propicia los conceptos clave en el análisis de fuerzas que actúan y afectan el currículo normalista. Tales conceptos de la genealogía corresponden a la emergencia y procedencia. Esta lógica analítica de la genealogía aborda el estudio de la historia, no desde el enfoque tradicional, sino desde el historiográfico: alejado de la tradición, la linealidad y la búsqueda de una verdad única. Se entiende que Foucault (2019) concibe la genealogía como una manera de hacer historia, donde el sentido historiográfico se vierte como una lógica potente de análisis de acontecimientos o sucesos. Para el filósofo francés, suceso o acontecimiento se refiere, no a las fuerzas presentes en la historia como eventos de gran significado, de fechas fijas, con héroes o caudillos, sino a fuerzas fortuitas que no obedecen a un destino. Desde esta perspectiva, la lógica analítica ayuda a comprender que el sentido histórico admite la existencia de una serie de acontecimientos accidentales que conducen a ausencias o discontinuidades en torno a un suceso.

Foucault (2019) considera que la genealogía reconoce como objeto dos nociones de Nietzsche: la procedencia y la emergencia. La primera explica una singularidad de eventos que han tenido lugar alrededor de determinado concepto y cuya formación ocurre en circunstancias de mediación, causalidad u oposición. Así, la procedencia reside en aceptar que somos resultado de accidentes, errores o complejas repeticiones. En tanto la noción de emergencia se concibe como el punto de surgimiento o ley singular de aparición, que se produce en un determinado estado de fuerzas. Su análisis tiene como propósito evidenciar de qué manera se produce el conflicto entre tales fuerzas o de qué manera tales fuerzas enfrentan la adversidad, se anulan o se revitalizan.

Bajo el horizonte teórico de la genealogía foucaultiana, se pretenden evidenciar los acontecimientos que mediaron para que en los planes normalistas emergiera o se relegara la asignatura de Inglés. En otras palabras, la genealogía se utiliza como instrumento analítico, útil para "percibir la singularidad de los sucesos, [...] reencontrar las diferentes escenas en las que han jugado diferentes papeles; definir incluso el punto de ausencia o el momento en que no han tenido lugar” (Foucault, 2019, p.8). Una vez delimitado el posicionamiento teórico, ahora es preciso poner en perspectiva el elemento articulador de lo histórico y educativo, que ordena el documento y que ha sido el hilo conductor del análisis: las reformas curriculares. 


\section{ESTUDIOS Y E N A Y OS}

Por más de un siglo se han efectuado reformas a los planes curriculares de las escuelas normales de México, algunos con y otros sin el inglés en la malla de asignaturas. En este documento se retoman diez reformas consultadas, que fueron objeto de un análisis documental, desde la época posterior a la independencia, con la escuela Lancasteriana, hasta 2018, con la Estrategia Nacional para las Escuelas Normales. Las diez reformas analizadas fueron a) México posindependencia, b) la institucionalización de las escuelas normales de México, c) los Congresos de Instrucción Pública I y II, d) la educación socialista, e) la unidad nacional y el Plan de 11 años e industrialización, f) el cambio a educación superior, g) la modernidad, h) el regreso del inglés al currículo normalista con la alternancia partidista, i) una reforma educativa controvertida, y finalmente, j) la incertidumbre actual.

Igualmente, estas reformas se acompañan de una figura metafórica que se explica a continuación.

\subsection{Ciclos ondulatorios: cimas y valles}

Apelando a una forma historiográfica de hacer una revisión de las reformas normalistas y de comprender los momentos en que emerge la asignatura de Inglés en los planes curriculares de las escuelas normales, se acude a una imagen de la física perteneciente a los movimientos ondulatorios. Dicha imagen concuerda con la no linealidad, las ausencias y discontinuidades de la genealogía. Esta metáfora ilustra los ciclos de altibajos que han involucrado al inglés en las escuelas normales. Se concibe la imagen de ciclo conformado por dos picos: uno alto, llamado «cima», y uno bajo, llamado «valle». Ambos picos ayudan a dimensionar las fluctuaciones que representan «la inclusión o no inclusión» de la asignatura de inglés dentro de los planes de estudio de las licenciaturas en las escuelas normales.

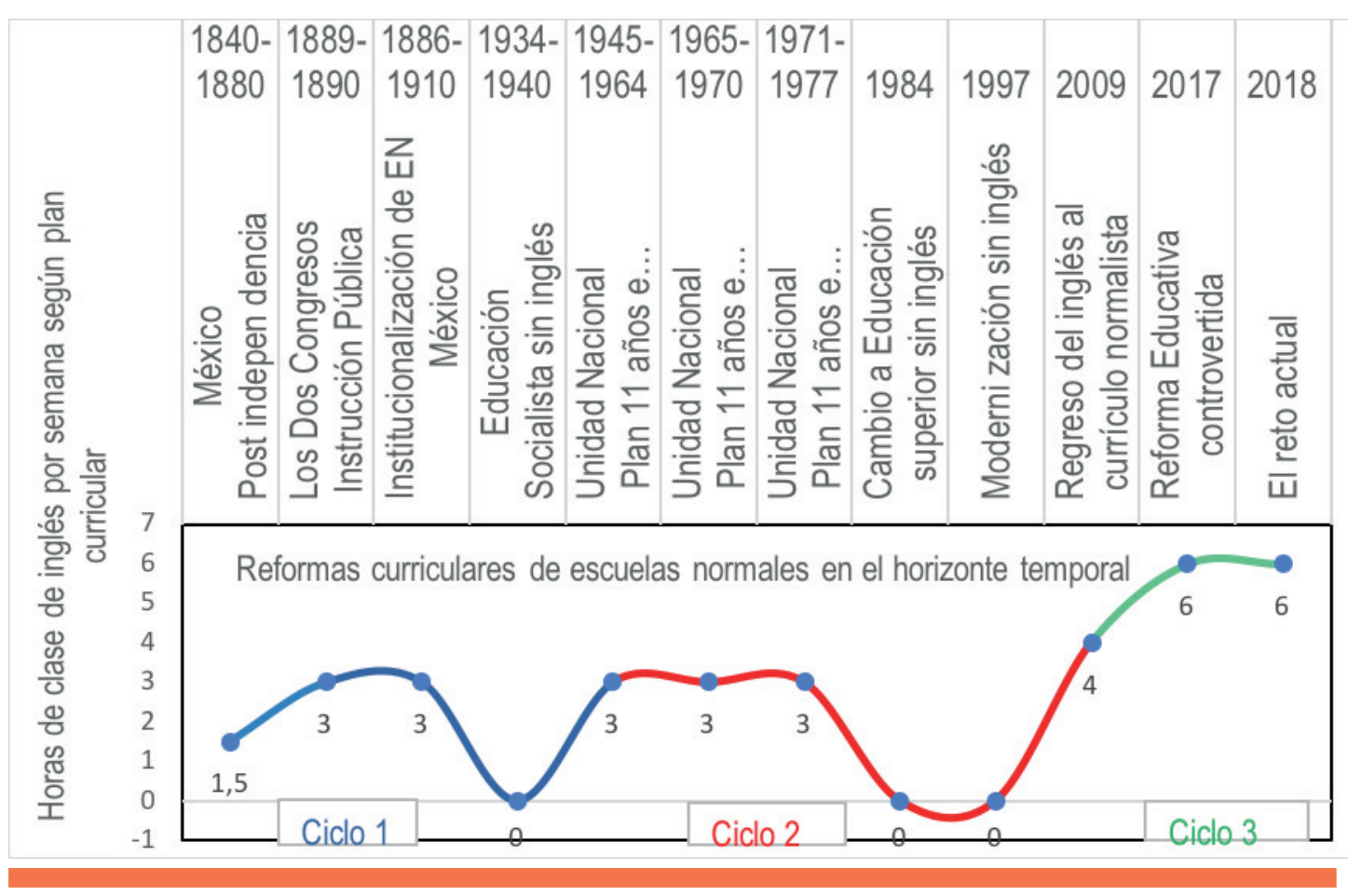

Gráfica 1. Ciclos de emergencia de la asignatura de Inglés en las reformas normalistas de México. Fuente: Elaboración propia 


\section{E S T U D O S Y E N A Y O S}

En la gráfica 1 se aplica la analítica genealógica en la disposición de los sucesos reformistas que dibujan un relieve repetitivo, ondulante y no lineal. Con ella se revelan las huellas que la asignatura de Inglés ha impreso a lo largo de las reformas normalistas. En el plano se aprecian el eje de horas de clase impartidas por semana, según cada plan curricular, contra el eje reformas curriculares de escuela normalista en un horizonte temporal. Esta figura representa un panorama general de los tránsitos por décadas y luego por sexenios de las reformas expedidas para la escuela de formación docente mexicana. En ella se delinean dos ciclos completos con sus respectivas cimas y valles y uno incompleto en el que apenas recorre la mitad de una cima. Más adelante, en este artículo, se exponen y se discuten cada uno de los ciclos, sus reformas con su expresión a favor o en contra de la inclusión del inglés en las licenciaturas, asimismo, las fuerzas socioeconómicas o políticas que convergen en las decisiones curriculares.

Tabla 1. Ciclos y sus correspondientes reformas con intensidad horaria

\begin{tabular}{|c|c|c|}
\hline Ciclos & Reformas que integran cada ciclo & $\begin{array}{l}\text { Horas de inglés } \\
\text { semanales }\end{array}$ \\
\hline $\begin{array}{l}\text { Ciclo } 1 \text { o de } \\
\text { emergencia }\end{array}$ & $\begin{array}{l}\text { a) México posindependencia } \\
\text { b) la institucionalización de las escuelas normales de México y } \\
\text { c) los Congresos de Instrucción Pública I y II } \\
\text { d) la educación socialista }\end{array}$ & $\begin{array}{l}3 \text { horas entre inglés y } \\
\text { francés } \\
3 \text { horas } \\
\text { Sin horas }\end{array}$ \\
\hline $\begin{array}{l}\text { Ciclo } 2 \text { o de } \\
\text { expansión y } \\
\text { contracción }\end{array}$ & $\begin{array}{l}\text { e) la unidad nacional y el Plan de } 11 \text { años e industrialización } \\
\text { f) el cambio a educación superior } \\
\text { g) la modernidad }\end{array}$ & $\begin{array}{l}3 \text { horas } \\
\text { Sin horas } \\
\text { Sin horas }\end{array}$ \\
\hline $\begin{array}{l}\text { Ciclo } 3 \text { o } \\
\text { de reciente } \\
\text { expansión }\end{array}$ & $\begin{array}{l}\text { h) la alternancia partidista y el regreso del inglés al currículo normalista } \\
\text { i) una reforma educativa controvertida } \\
\text { j) la incertidumbre actual }\end{array}$ & $\begin{array}{l}4 \text { horas } \\
6 \text { horas } \\
6 \text { horas }\end{array}$ \\
\hline
\end{tabular}

Fuente: Elaboración propia

La tabla 1 muestra la articulación de los ciclos, las reformas y la intensidad horaria indicada para el inglés. La composición de cada ciclo concuerda con tres reformas. El ciclo 1 se denomina de emergencia, puesto que congrega los primeros indicios de enseñanza de idiomas, la reforma de institucionalización normalista con los Congresos de Instrucción Pública al coincidir en temporalidad y en propósito y la educación socialista. El ciclo 2 es de expansión y contracción incluye los cambios durante la unidad nacional, el Plan de 11 años e industrialización, el giro hacia la educación superior y el rumbo hacia la modernidad. Por último, el naciente ciclo 3 o de reciente expansión recoge las reformas de la alternancia partidista, el regreso del inglés al currículo normalista y la época actual.

\section{CICLO 1. DE EMERGENCIA, 1840-1940}

\subsection{México colonial y posindependencia}

En la época colonial, la educación era destinada a los descendientes de familias extranjeras o criollas de alta posición socioeconómica. Ciertas artes, habilidades y saberes eran inculcados 
por profesores formados en otros países o por profesores extranjeros contratados de manera particular. En concordancia con lo anterior, esta modalidad educativa individualista tuvo mucha difusión en la élite (Bazánt, 1993; Mendoza, 2017), debido a la proximidad con la cultura del viejo mundo. De acuerdo con Aguirre (2010), entre las habilidades enseñadas se encontraban los idiomas, pues eran parte de las artes y conocimientos del imaginario social de la época.

Al terminar la guerra independentista, el interés de los ayuntamientos en México radicó en intentar proveer educación básica y formar docentes (Staples, 1992). Las escuelas de primeras letras y las escuelas normales estaban ligadas. Una vez aprobado el curso de lectura y escritura, los estudiantes se convertían en aprendices, quienes eventualmente, tras seis meses de formación, procedían a ser profesores (Estrada, 1977). Cabe recalcar que en las escuelas de primeras letras no se enseñaba ningún idioma. Así, con el paso del tiempo, la falta de recursos de cada ayuntamiento complicó que estas escuelas se pudieran sostener.

La difusión de la educación normal dio un giro con la Compañía Lancasteriana, que recibió el apoyo gubernamental al ser la primera iniciativa de orden nacional para la formación de maestros entre 1842 y 1870 (García, 2015). Una vez obtenido el aval presidencial, la Compañía Lancasteriana se constituyó en la forma de instrucción predominante por casi cuatro décadas. García (2015) explica que, con la llegada de otras modalidades educativas, se hicieron tangibles una variedad de detracciones sobre la disciplina casi de milicia, la memorística, la debilidad pedagógica de los monitores, contra la rápida expansión de docentes y escuelas para estudiantes del sector popular. Al respecto, Quiñones (2001) señala que los estudiantes más avanzados o monitores debían llegar con tres horas de anticipación para ser instruidos en las clases del día y que durante tres días a la semana, entre las tres y las cuatro de la tarde, impartían clases de idiomas, con apoyo del libro de inglés de Robertson o de francés de Ollendarf.

Siguiendo el marco analítico de la procedencia de Foucault (2019), en el que suceden desajustes o repetitividad de condiciones, se incrusta la reproducción de patrones europeos en la conformación histórica mexicana de la enseñanza de las lenguas. Sus concepciones repetían dichos patrones que entrañaban varios sentidos: uno civilizatorio, otro de paridad con las culturas dominantes y uno más de supervivencia académica. Estos sucesos dan cuenta de la relevancia de los idiomas en la noción de formación de futuros profesores de instrucción elemental.

A pesar de encontrarse en un momento disímil de evolución educativa frente al contexto europeo, se entroniza en el contexto mexicano una identidad educativa distinta. Es decir, la naturaleza diversa de un México con tanto desbalance educativo desborda hacia una incidencia europea en el currículo, que legitima un círculo de dominación entre pioneros y autoridades educativas, lo que se inscribe y cumple con lo que Foucault (2019) plantea como el propósito del trabajo genealógico: discernir el dominio en la historia.

\subsection{La institucionalización normalista, el porfiriato y los congresos de instrucción pública}

Dos de los críticos de la escuela lancasteriana fueron Enrique Laubscher y Enrique C. Rébsamen. Enrique Laubscher — pedagogo, natural de Baviera, discípulo de Froebel— estudió la pedagogía y las humanidades de la época y ejerció el magisterio en su país. En 1880, se traslada a México y 
tres años más tarde funda la Escuela Modelo de Orizaba, donde impartió un nuevo método para profesores de instrucción elemental, cuyas asignaturas son enumeradas por Ramos (1977): “el plan de estudios comprendía: lenguaje, cálculo, geometría, dibujo, geografía, historia, ciencias naturales, inglés, francés, moral, música y gimnasia” (p.74). En este modelo, tanto el inglés como el francés figuran con un espacio curricular en igualdad temporal para su enseñanza.

Con esta propuesta, el pedagogo alemán se convierte en un pionero educativo. En poco tiempo, logra instalar sus concepciones y experiencias aprendidas en Europa en un ámbito educativo en déficit de conocimientos y destrezas pedagógicas, como era el caso de México en ese entonces. Ante la necesidad de textos, Laubscher realiza traducciones al español de libros de primer año de inglés y francés para sus clases (Larios, Hernández y Coronado, 2016).

De manera similar, Enrique Rébsamen llega a México — procedente de Suiza - una vez que concluye sus estudios avanzados en educación, con experiencia tanto de docente en idiomas (inglés, alemán, francés, latín) como de director de una escuela normal. Su primer desempeño en México fue como maestro de francés para una familia conservadora de Nuevo León, pero sus desacuerdos hicieron que abandonara este trabajo. Luego se muda a Veracruz y se une a Laubscher para trabajar en la Escuela Modelo de Orizaba. Posteriormente, en 1886, funda la Escuela Normal de Xalapa. Su pensamiento sobre el deber ser de la educación lo lleva a concretar la conformación de un sistema educativo para México (Vera, 2006). Su perspectiva sobre el currículo para ser maestro de primaria elemental y superior era una selección de asignaturas tanto científicas como de aplicación práctica, con una duración de cinco años si se deseaba completar la formación. Al respecto, Murillo (1974, citado en Vera, 2006), en su descripción de las 45 asignaturas de esa época, enuncia que para primer y segundo grados se impartían clases de francés; y para tercero y cuarto grados, clases de inglés. Las clases tenían una intensidad horaria de tres horas semanales.

En 1887, mediante ley presidencial, se crea en la capital mexicana la Escuela Normal Nacional para Varones y al año siguiente la Normal de Mujeres, pues ya se sabía de diferentes iniciativas en otros estados (Ducoing, 2004). El programa de asignaturas replicó el paradigma positivista de la época al orientarlo a la cientificidad y experimentación. De acuerdo con un estudio de corte histórico (Meneses, 1973), el plan normalista se integraba de 21 asignaturas, entre ellas Inglés y Francés. Esto indica una concordancia de programas en las nacientes e influyentes escuelas normales.

También las escuelas normales privadas en sus inicios incorporan el idioma inglés. Los misioneros protestantes venidos de los Estados Unidos de Norteamérica traen consigo, además de su intención religiosa, su lengua nativa y, eventualmente, esto tiene gran impacto en el sector educativo. En su proceso evangelizador, era bien visto que los pastores aprendieran el español y que a la vez enseñaran la lengua anglosajona para la lectura de sus textos y los servicios religiosos. Así sus feligreses dominaban el idioma y el rito religioso. De acuerdo con Rodríguez y Romero (2018), los misioneros de la Iglesia Metodista Episcopal se instalan en el norte y centro del país entre 1873 y 1878. Publican revistas y promueven servicios de habla inglesa; entre sus obras destacan centros de estudio religioso y de carácter normalista, que luego transformarían en escuelas normales y universidades

Es conocido que, en esta época de finales del siglo XIX, una marcada preponderancia francesa impera en México y su idioma tiene mayor relevancia frente a otros en los años de instrucción 
elemental. El francés se enseñaba durante los tres años del curso, mientras que el inglés contaba con dos años de dedicación formal. Parte de esa tendencia afrancesada se debía a dos causas: a) dos de las normales prominentes, la de Orizaba y la Veracruzana, habían sido orientadas por extranjeros cuyos sistemas se centraron en la construcción de una normatividad educativa a gran escala. b) El primer Congreso de Instrucción Pública había sido acompañado por las ideas de Jules Ferry, exministro de educación galo, quien en 1880 organizó un congreso educativo en su país, prestando la idea de uniformidad a la versión mexicana (Ballín, 2007). Sin embargo, otros sectores sociales y económicos también recibieron dicha dominancia.

El Porfiriato logró atraer la inversión europea y acabó aceptando el pago de la deuda exigida por Francia, luego del restablecimiento de relaciones diplomáticas en 1880. El desarrollismo, que duró hasta antes de la revolución, trajo consigo "una impresionante penetración económica y cultural” (Meyer, 2011, p.15), que se resentía en el día a día, tanto por las familias acomodadas en sus costumbres como por la población en su educación. El Porfiriato fue condescendiente con una oleada económica francesa (extracción, banca, comercio, industria) que enriqueció a quienes ya estaban instalados en México y promovió el afrancesamiento cultural.

Aunque ya la época colonial había cesado, se advierte una marcada dominación cultural, económica y política de Europa y Norteamérica durante el gobierno de Porfirio Díaz. La treintena de años gobernados por Díaz trajo prebendas a industriales extranjeros en desmedro de las condiciones laborales de campesinos y obreros. El surgimiento de una élite política, económica y religiosa dominadora de las condiciones forjó la desigualdad social y educativa.

El gobierno porfirista alcanzó niveles dictatoriales al coartar la libre expresión de la prensa y cualquier expresión civil de descontento laboral por medio de la represión militar. Estas condiciones detonaron la sublevación que se convirtió en la Revolución mexicana. Una revolución que por diez años tuvo consecuencias de pérdidas humanas y económicas. La muerte de casi un millón de personas tuvo repercusiones en la mano de obra, sobre todo en el sur del país.

En cuanto a lo económico, Womack (2012) sintetiza que México pasó por tres etapas: 1) etapa de dependencia del influjo internacional entre 1880 y 1910; 2) etapa de destrucción de la organización económica dependiente de Europa y Estados Unidos entre 1917 y 1920; y 3) etapa de crecimiento de 1921 a 1940 por la facilidad para las fuerzas productivas. Una consecuencia que cobró relevancia fue la Constitución de 1917, que reorientó la organización política, limitó la acción de los extranjeros, prohibió el monopolio y penalizó la no producción de propietarios. Paralelamente, abrió la puerta al socialismo.

\subsection{La educación socialista}

El periodo que sobrevino después de la Revolución mexicana trajo consigo una brecha en la educación. Es el momento político de Lázaro Cárdenas o del cardenismo (1934-1940). Esta época de gobierno cardenista corresponde con una Europa en tensión por la proximidad de un enfrentamiento bélico y con una Unión Americana cercana a convertirse en potencia mundial. El dominio sociopolítico cambia de extremo: del Porfiriato y su benevolencia a la injerencia extranjera, al cardenismo y su refundación mexicana. Este dominio nacionalista estableció un nuevo orden 
en el saber y en el hacer político-económico. En el saber, se expandieron las escuelas normales rurales y se apropiaron de saberes técnicos para el desarrollo comunitario. En el hacer político económico se promovieron cambios en la Constitución y en la producción industrial.

La reforma al sistema educativo fue uno de los pilares del proyecto político de Cárdenas, que se estableció al incluir en el artículo tercero de la Constitución elementos como la exclusión de la religión, el fanatismo y calificar en adelante la educación como «socialista». De acuerdo con Meneses (1998c), en el currículo normalista de la reforma de 1935 se propusieron asignaturas relacionadas con economía política y con los problemas políticos y económicos del país, economía doméstica, geografía económica, sociología de la educación, historia de la cultura y del movimiento obrero y literatura del proletariado. En general, estas asignaturas se fundamentaron en el método del materialismo dialéctico aplicado a la teoría marxista de la época.

Con respecto a este ciclo, el análisis genealógico permite entrever una lógica de causalidad sobre la ausencia de la asignatura de lengua inglesa en las escuelas normales mexicanas al descifrar la incidencia de la educación socialista y la proyección de la Unión Americana. El nuevo engranaje educativo socialista no admitió y excluyó por muchos años el inglés de las normales urbanas y rurales. Se entiende que el peso de un gobierno hegemónico de treinta años, como el de Porfirio Díaz con un enarbolado elitismo que recrudeció el abandono de obreros e indígenas y la consecuente lucha civil por el rescate del pueblo mexicano durante la Revolución mexicana condujeran a que el gobierno socialista electo se concentrara en el ideal del pueblo, que redundaría en una preferencia de las lenguas nativas sobre las extranjeras. Con respecto a la formación de fuerzas de contingencia sobre el devenir educativo, se conoce que los conflictos del gobierno cardenista con las petroleras anglosajonas interfirieron para que, en ese tiempo, las lenguas extranjeras se excluyeran en las instituciones del sistema educativo mexicano. Es así como los eventos políticos y de turbulencia económica confluyeron y restringieron la enseñanza del inglés en este periodo del cardenismo mexicano.

\section{CICLO 2. ENTRE LA EXPANSIÓN Y LA CONTRACCIÓN, 1940-1975}

\subsection{La unidad nacional, el plan de 11 años y la industrialización}

Pasados los acontecimientos que cambiaron el panorama político en el país, tales como la Primera Guerra Mundial, la Revolución mexicana y la época cardenista, sobrevino el periodo de la Unidad Nacional, en el que se retoma la enseñanza de los idiomas en México. El presidente Manuel Ávila Camacho (1940-1946) revierte la influencia cardenista en la educación al eliminar los enunciados socialistas de la Constitución nacional e introducir la industrialización. Un auge en la producción y exportación ayudó a engranar un ímpetu diferente. A esto se sumó el nombramiento de Jaime Torres Bodet como secretario de Educación Pública en 1944, en la que sería su primera asignación al frente de esta Secretaría. Entre sus acciones de política educativa, como nuevo secretario, Torres Bodet convocó al Congreso Nacional de Escuelas Normales e hizo la reforma de planes curriculares (Hurtado, 2002). En referencia a las asignaturas de idioma extranjeros, Meyer (2011) aclara que "el idioma francés estuvo aún presente en los planes de estudio entre 1940 y 1945” (p.17). Lo anterior debido, por un lado, a la situación de desventaja que Fran- 
cia padeció al haber sido invadida por la fuerza nazi, y después rescatada por los aliados, junto al general De Gaulle exiliado en Gran Bretaña. Por otro lado, el inglés y su influencia iban en aumento por la posición de Estados Unidos e Inglaterra al final de la Segunda Guerra Mundial.

El currículo normalista se unificó para las escuelas normales urbanas y rurales en 1945. Esto incluyó, de acuerdo con Rodríguez (2006), 24 materias básicas en las que se ofrecía escoger entre francés o inglés como idiomas extranjeros; 10 asignaturas de actividades de agricultura, artes, economía y cultura física; 22 materias profesionales como pedagogía, educación, psicología, entre otras; y siete de cultura general. En esta reforma educativa de gran envergadura, que transformó el sistema educativo mexicano, ya se empieza a notar una apertura hacia la especificidad del conocimiento con 63 asignaturas para la formación inicial. Si se revisa la disminución en el número de horas destinadas a los idiomas, se constata una ligera exclusión, pues se restringió a inscribirse a un solo idioma por elección, debido tal vez al aumento en la cantidad de asignaturas.

Los cambios efectuados en los programas de estudio en 1945 se mantuvieron por cerca de 20 años, y sin reestructuraciones. En 1954, el presidente Ruiz Cortínez (1952-1958) citó a una Junta Nacional de Normales para estudiar la deserción, especialización y diferenciación entre las normales urbana y rural; las sugerencias ahí generadas sirvieron posteriormente para la formación docente.

En el siguiente periodo sexenal, Adolfo López Mateos (1958-1964) designa nuevamente a Jaime Torres Bodet en la escena de la Secretaría de Educación Pública, quien presenta dos planes de estudios normalistas, uno en 1959 y otro en 1964. A pesar de que se generaron cambios en los programas, estos no afectaron la asignatura de idiomas; permaneció la opción de elegir entre francés o inglés. El plan curricular de 1964 comprendía cerca de 60 materias (incluidas las opcionales), entre las que permanecían los idiomas, que por primera vez se nombraron como Lengua Extranjera (traducción I-II-III), de quinto a séptimo semestre, y como opcional en octavo semestre (traducción IV) (Meneses, 1991).

En 1975, el Consejo Nacional Técnico de la Educación cita a asamblea, y tras varias reuniones se produce un nuevo plan de estudios que abandona los análisis teóricos de orden sociológico e histórico (Figueroa, 2000) y se inserta más en lo técnico. No obstante, se reestructura con la eliminación de las didácticas y se escogen algunas clases con práctica pedagógica para un total de 76 materias. En primer y segundo semestres se situarían Inglés I e Inglés II, respectivamente (Meneses, 1991). Se hizo una reducción a la mitad en el número de semestres que tenía un curso de Enseñanza de Lengua Extranjera con respecto al plan de estudios de 1972-1973 que tenía cuatro semestres con 110 asignaturas. Es preciso detallar que en el plan de 1975 se nombró la asignatura como “Inglés”, y desapareció totalmente la opción de aprender el francés de la formación normalista.

Este cambio de denominación de “Lengua Extranjera” en el currículo de 1964 a “Inglés” en 1975 y la eliminación del Francés del currículo generaron una nueva configuración, pues al no especificar las lenguas que se enseñarían, se aceptó la aparición de otros idiomas alternativos —antes no enseñados - que por circunstancias económicas — como la llegada de la industria automotriz alemana - se integraron al grupo que antes conformaban únicamente el francés y el inglés.

Con estas acciones en política educativa se fue configurando una tendencia minimizadora con respecto a la importancia de las lenguas en el currículo normalista, que desde 1970 — después de 
los movimientos estudiantiles y magisteriales - le dieron otra visión a la carrera docente: más conocimiento y menos formación para la docencia. Se puede afirmar que se afianzó una lógica utilitarista en las escuelas normales con estos cambios. En otras palabras, se fue desarrollando una tendencia a la práctica de tipo inmediatista, evidenciada en que, con poco tiempo de estudio, tan solo tres años de ciclo formativo, inmediatamente había la posibilidad de "conseguir una plaza segura de trabajo" (Yurén, 2009, p.41).

\subsection{El cambio a educación superior}

La reforma de 1984 tuvo como piedra angular el propósito de elevar a la categoría de licenciatura a los programas de las escuelas normales de México. Esto se consideró como otorgar un reconocimiento a la profesión docente y posicionar los programas a nivel de educación superior. Este cambio curricular abarcó varios giros. Uno de ellos fue el tiempo de estudios de la licenciatura, que cambió a ocho semestres; además, el plan unió dos áreas de formación: tronco común y especialidad; el tronco común se dividió en cuatro líneas de formación: instrumental, pedagógica, psicológica y social; en la especialidad se incorporaron más asignaturas, según la licenciatura, con un número de asignaturas entre 62 y 70 (acuerdo $\mathrm{N}^{\circ} 134,1988$ ). Estas áreas no incorporaron la asignatura de Inglés para su enseñanza, a pesar de que en el nivel superior es necesaria la comunicación y difusión de investigación, de acciones de docencia y de servicio.

El tránsito de las escuelas normales hacia la educación superior fue un logro esperado por mucho tiempo. Este paso a la educación superior llevó a que los estudiantes normalistas lograran ser considerados licenciados, para lo cual el requisito era haber cursado la preparatoria pedagógica (Navarrete, 2015; Martínez y Contreras, 2016). Al adquirir el nivel terciario, las escuelas normales asumieron la tarea de desarrollar prácticas de docencia, investigación y difusión cultural (Medrano, Méndez y Morales, 2017). Sin embargo, de manera inesperada, esta reforma curricular anuló del plan de estudios la asignatura de Inglés, a pesar de estar bajo el influjo de una creciente apertura económica, de una expansión en conocimiento y comunicaciones. La tendencia de conflicto entre escuela normal y dinámica económica emerge desde el condicionamiento de los estudiantes para su privación de un referente socio-económico en un ambiente que requería la habilidad de la lengua extranjera, por la cercanía geográfica con países angloparlantes (al norte con Estados Unidos y al sur con Belice), además por lo que se perfilaría en el futuro como la era de la globalización.

\subsection{La modernidad sin inglés en el currículo normalista}

A partir de la reforma de 1997 al cúrrículo de las escuelas normales implementaron cuatro líneas de trabajo inscritas en un marco llamado Programa para la Transformación y el Fortalecimiento Académicos de las Escuelas Normales. Las cuatro líneas consideraban algunos planes curriculares, a los que se les añadieron criterios y orientaciones sobre el proceso de enseñanza y aprendizaje, un programa de mejoramiento y actualización docente, algunas directrices de gestión para las escuelas normales y las adecuaciones en la planta física, tanto en materiales y recursos como en reparación y ampliaciones de las instalaciones. La malla curricular de este plan de 1997 agrupaba tres áreas de actividades (SEP, 2002). 
Por primera vez el programa para la Transformación y el Fortalecimiento Académicos de las Escuelas Normales desglosa una serie de rasgos que comprometen habilidades intelectuales, dominio de las actividades didácticas, competencias didácticas, identidad profesional, capacidad de respuesta a las condiciones sociales (SEP, 2002). El número de asignaturas se redujo a 45, entre las que no se integraba la lengua extranjera anglosajona.

Dos gobiernos seguidos de apertura económica encaminaron al México de fin del siglo XX hacia un paradigma económico controversial. Con la entrada en vigor de la Reforma de Modernización Educativa de 1992, se sublima la triada del Estado, sector económico y sector educativo que luego adquirió cuerpo físico al establecer como objetivo de la educación en su periodo: "poner en sintonía la educación con el sector productivo, con lo cual se problematiza la formación docente", como lo afirma Ofelia Cruz (2007).

A pesar de que, en la reforma de 1997, para la transformación y fortalecimiento de las escuelas normales de México se nota una ausencia de asignaturas de lenguas extranjeras dentro del plan de estudios, hubo algunos estados e instituciones normalistas que, por voluntad de directivos, docentes y estudiantes, abrieron cursos extracurriculares de inglés, según lo enuncian Salazar, Chapa y Cantillo (2017). Este acontecimiento revela con claridad que desde el gobierno estatal se promovía una nulidad hacia el aprendizaje de las habilidades comunicativas en Inglés, necesarias para un trayecto de futuro desempeño, de especialidad y de nuevas capacidades; en tanto que esa normatividad social y pedagógica repercutió en prácticas docentes que "legitiman intereses y acciones sociales particulares, y al mismo tiempo omiten otras posibilidades" (BarrónTirado y Pontón-Ramos, 2013, p.204). Así se forjó una ausencia de conocimiento que excluyó a muchas generaciones de egresados de las oportunidades meritorias, que veinte años más tarde se constituyeron en dificultades económicas y laborales.

Esta no inclusión del inglés en la malla curricular de 1997 se instaura pese a la firma del Tratado de Libre Comercio de América del Norte (TLCAN). Este acuerdo económico fija la bandera de la competitividad y del bienestar para los pueblos de los países firmantes: México, Estados Unidos y Canadá. Empero, desde la autoridad educativa, se obvia la conexión entre la educación y la economía y se repite una dinámica que pareciera que estos factores no pudieran coexistir — como en la época socialista-, cuando lo que se vivencia es la apertura económica al capitalismo neoliberal.

\section{CICLO DE EXPANSIÓN RECIENTE 2006-2018}

\subsection{El regreso del inglés al currículo normalista con la alternancia partidista}

El segundo mandato presidencial del Partido Acción Nacional, presidido por Felipe Calderón Hinojosa (2006-2012), apeló a alianzas partidistas para ratificar sus propuestas programáticas, igual que en los dos anteriores sexenios. A diferencia de su antecesor, Vicente Fox Quesada (2000-2006), quien cerró escuelas rurales y disminuyó el acceso a las escuelas normales, Calderón Hinojosa promovió la construcción de Centros de Formación e Investigación Normalista y llamó a un cambio de planes curriculares en 2009. Este nuevo plan buscó incidir en la flexibilidad a través de la incorporación de la lengua adicional (inglés en este caso) y la tecnología de la información y la comunicación, en aras de lograr el contacto con el mundo globalizado por medio de la adquisición de competencias y destrezas. 
Con un retraso de tres años, la implementación de los planes 2012 llegó a las escuelas normales con sus respectivos materiales. Para este cambio, se consideraron 55 asignaturas de 291 créditos, agrupadas en cinco trayectos formativos: 1) Psicopedagógico, 2) Preparación para la enseñanza y el aprendizaje, 3) Lengua adicional y Tecnología, 4) Optativos y 5) Práctica Profesional (DGESPE, 2012). De acuerdo con la malla curricular, en el trayecto formativo de Lengua adicional y Tecnología se imparten siete asignaturas de cuatro horas semanales, con valor de 4.5 créditos cada una, desde el primero hasta el séptimo semestres. Es decir, en primer y segundo semestres aquellas relacionadas con las tecnologías de la información y de tercero a séptimo semestres las de los niveles de Inglés A1, A2, B1, B2 que se traducen de un nivel principiante a nivel intermedio alto.

Es necesario discutir que en la malla curricular 2012 de las escuelas normales mexicanas aparece el concepto «lengua adicional» para denotar la asignatura que desde el plan curricular de 1964 era llamada lengua extranjera. Desde la subdisciplina de la lingüística aplicada, la denominación de lengua adicional se concentra para aquellas lenguas que son no nativas, que son el medio de instrucción y alfabetización académica, que se hablan en el salón de clase y en las instancias educativas, que requieren apoyo para acceder al currículo, que tienen un soporte institucional de recursos para aprendices, que orientan actividades en casa para su exposición continua a través de un modelo lingüístico, que monitorea e interviene en caso de bajo desempeño del estudiante (MacLean, 2011).

En efecto, este constructo llamado «Lengua adicional» tiene aplicación como política de lengua en el Reino Unido. En pocas palabras, se refiere al inglés que se enseña a migrantes recién llegados, quienes incluso tienen dos o más lenguas aprendidas en casa y que califican para acceder a tutores y recursos de apoyo (Baker, 2001). Sin embargo, este no es el caso de las escuelas normales mexicanas. En atención a varias características, el español es el idioma de instrucción en las licenciaturas, con el que se gestiona el ambiente escolar. De esta manera, el inglés, en la reforma 2012, se anexaba de nuevo a los planes curriculares, debido a lo cual no existían programas de apoyo ni modelo lingüístico para los hogares. Por lo anterior, el término «Lengua adicional» dibuja una imagen errada, poco consecuente con el momento histórico y refleja transferencias de políticas poco documentadas.

Como se mencionó, en 2006, la elección por segunda vez de un gobierno panista a la presidencia, con Felipe Calderón Hinojosa, abrió la puerta para el regreso del inglés al currículo normalista, que estuvo influenciado por condiciones político-económicas particulares. A tan solo cuatro meses de su posesión como presidente, se estableció un acuerdo con Estados Unidos para la acción en contra del narcotráfico, esto se denominó «Iniciativa Mérida». Este acuerdo establecía presupuesto, equipos y asesoría para la lucha antidrogas, similar al Plan Colombia, sin la presencia militar especializada en campo, sobre lo cual Ortega y Somuano (2015) aducen que fue una meta poco factible.

Un acontecimiento que tuvo repercusiones en muchos aspectos de las situaciones en México, durante ese sexenio (2006-2012), fue sin duda la recesión económica de 2008, debido a la proximidad geográfica y económica con los Estados Unidos. El modelo de exportaciones al país del norte contrajo las fuerzas internas mexicanas a causa del TLCAN, firmado en la última década del siglo XX, combinado con un discurso en el que prevalecía el llamado a incorporarse a un mundo globalizado en pro de la «competitividad». De acuerdo con Meyer (2015), estas con- 
diciones provocaron un aumento en la migración hacia Norteamérica, que llegó incluso a ser de un 10\% de la población, lo que equivale a cerca de 12 millones de mexicanos en Estados Unidos.

\subsection{El regreso del partido hegemónico y la reforma normalista de 2017}

Durante el debatido gobierno de Enrique Peña Nieto (2012-2018) en materia educativa, el detonante hacia la promoción del inglés en las escuelas normales fue el lanzamiento del Modelo para Escuelas Normales y su Estrategia de Aprender Inglés (SEP, 2017). Esta propuesta marcó dos direcciones: docencia y curricular. Con respecto a la docencia, 546 docentes fueron contratados con plaza federal fija y nivel salarial más alto (SEP, 2018). Con miras a mejorar las condiciones de enseñanza del inglés en las escuelas normales, los docentes debieron acreditar certificados internacionales en docencia y proficiencia del idioma avalados por el Concejo Británico.

En lo curricular, se elevó a seis horas las clases de inglés, desde el semestre primero al sexto, con valor de 6.75 créditos en todas las licenciaturas de las escuelas normales (excepto aquellas escuelas normales en lenguas indígenas). La segunda medida fue la propuesta de creación de la licenciatura en Inglés. Y la tercera fue la certificación para estudiantes de último año en nivel usuario independiente. Estas dos últimas no se concretaron a razón del poco tiempo restante para su implementación, de las reacciones y resistencias normalistas a la reforma.

En un ámbito social y económico agitado se desarrolla este periodo de relevancia para el idioma inglés en las escuelas normales. En mayo de 2017 se inician las rondas de negociación del segundo Tratado de Comercio de América del Norte (T-MEC) con un recién electo presidente en Estados Unidos que agita la dinámica económica y migratoria entre México y Estados Unidos y que reclama mejorar las condiciones resultantes para la Unión Americana. Esto se traduce en exigencias para México en términos de formación laboral y remuneración de alto nivel. Simultáneamente, se lanza la reforma normalista en 2017, y en mayo de 2018 se contratan a los nuevos docentes de Inglés categoría C. En noviembre de 2018 se firma el T-MEC. En esta ocasión, se vislumbra una conexión robusta entre la economía y la educación, a diferencia de lo ocurrido en la reforma de 1997 durante la firma del TLCAN. En este sentido, se responde a una dinámica emparentada entre formación y competitividad que influye en la revitalización del inglés en la malla curricular de las escuelas normales.

En este ciclo, el enfrentamiento entre diversas fuerzas sociales y económicas ha generado en varios sexenios su recuperación hacia una expansión de las necesidades de aprender inglés, como en el caso del segundo y tercer ciclo (Unidad Nacional, los 11 años y la industrialización), durante los cuales el inglés tuvo un lugar en los planes de estudio normalistas. En el ciclo de reciente expansión 2009-2018, las iniciativas a favor del aprendizaje del inglés volvieron a revitalizarse con el apoyo de algunas organizaciones privadas, que se enfrentaron con las fuerzas educativas tradicionales y en esa discusión salieron fortalecidas las fuerzas económicas para orientar la reforma que detuvo una discontinuidad de más de 30 años del inglés curricular en las escuelas normales.

\subsection{La incertidumbre actual y la abrogación de la reforma anterior}

En 2018, la elección de un gobierno con tendencia antineoliberal, cuya propuesta de campaña se centró en la abrogación de la reforma educativa anterior, derivó en incertidumbre. Las medidas 
adoptadas en política educativa remiten a alianzas con los gremios sindicales tradicionales, siguiendo los pasos de gobiernos neoliberales como el de 1988, 2000 o 2006. No obstante, se han adoptado sin oposición los planes curriculares 2018, que incluyen el inglés y se continúa con la implementación de la «Estrategia nacional de inglés para las escuelas normales».

Así, el actual gobierno (2018-2024) de Andrés Manuel López Obrador trae una consigna hacia la abrogación de las acciones en política educativa, hechas durante el periodo ejecutivo anterior (2012-2018). Esto plantea severos interrogantes sobre la magnitud de las consecuencias en los órdenes legal, legislativo, laboral, educativo, económico y social. Sin embargo, las escuelas normales aún no están en el centro de las transformaciones educativas, por lo que continúa siendo efectivo el plan curricular 2018 para la asignatura de Inglés, asimismo los nombramientos en las plazas federales de los docentes de Inglés de la reforma. Por el momento, se señala que este periodo aparece como detenido en la temporalidad, provocando preguntas y dudas sobre las condiciones en las escuelas normales, con referencia a la austeridad manifiesta desde el poder ejecutivo.

Se hace oportuno mencionar que, al momento de la redacción de este artículo, se vive una contingencia sanitaria de orden mundial, que ha llevado a la virtualidad al sistema educativo por completo. Esto ha significado un reto en aspectos pedagógicos por ser un suceso de aislamiento social inesperado. De esta manera, han sucedido en el proceso situaciones difíciles para el aprendizaje, que manifiestan condiciones pedagógicas por resolver en el corto plazo. En lo tecnológico, el desbalance en la disposición de los recursos para atender la gran masa estudiantil. En el aspecto burocrático, un énfasis en la exigencia de evidencias desde las jerarquías educativas con el fin de mantener el control sobre docentes y estudiantes.

Con lo anterior se reitera que, ante la menor circunstancia inédita, se repiten los esquemas tradicionales de inequidad y control, aun cuando el contexto supone otros vínculos y otras lógicas de trabajo escolar. A pesar de todo lo anterior, aún se sigue a la espera de los cambios educativos que este gobierno aplicará, debido a que muchas de las instancias y dependencias educativas se encuentran trabajando desde la virtualidad, en espera de la reactivación social y regreso a la presencialidad educativa. La incertidumbre desde lo social, sanitario, político y económico hace parte de esta espera sobre las acciones político-educativas de este gobierno que genera algunos cuestionamientos, por ejemplo, ¿qué repeticiones, eliminaciones o revitalizaciones operarán durante este sexenio?

\section{REFLEXIÓN FINAL}

La reconstrucción historiográfica ha logrado pormenorizar ciclos de inclusión y no inclusión de las lenguas extranjeras en el currículo de las escuelas normales. Estos ciclos ondulantes son espejo de las condiciones que la lengua extranjera ha trasegado para convertirse en asignatura oficialmente aceptada en los currículos normalistas. Se han evidenciado dos ciclos completos y uno que está en plena formación. La duración de los ciclos varía de manera considerable: el primero o de emergencia va de 1840 a 1940 (se mantiene aproximadamente un siglo); el segundo o de expansión y contracción de 1940 a 1997 (sesenta años); y el tercero o de reciente expansión viene desde 2006, inició ascenso en 2017 y se ha mantenido por tres años. 
Asimismo, los altibajos hacen tangibles cimas, valles, amplitudes y extensiones. Se aprecian tres cimas o picos altos: 1) la reforma en que se institucionaliza la escuela normalista con los Congresos de Instrucción I y II bajo el gobierno porfirista. 2) Las reformas entre la Unidad Nacional, la reforma de los 11 años y la industrialización. 3) La reforma 2018 y el momento actual. Las tres se diferencian por el número de horas a la semana dedicadas al inglés, según se indica en cada plan. Debieron pasar 120 años para que se incrementara una hora desde el ciclo 1 hasta el 3 al pasar de tres horas semanales a cuatro; mientras que transcurridos 12 años del ciclo 3 se aumentó dos, que equivale pasar a seis horas semanales.

Al respecto, se analiza que el discurso inicial de la lengua extranjera provino de una educación más ilustrada bajo entornos europeos dominantes. Seguidamente, hubo momentos de inclusión del inglés en el currículo, que coinciden en expansión social e injerencia internacional durante los periodos de Porfirio Díaz, de la Unidad Nacional y la retoma del poder por el partido hegemónico en 2012. De acuerdo con la analítica genealógica de Foucault, la inclusión del inglés procede de circunstancias de mediación económica mercantilista derivadas del influjo tecnológico industrial en los tres periodos. Siendo esas mediaciones: la construcción y el transporte en el Porfiriato, la llegada de las industrias inversionistas en la Unidad Nacional y la ratificación de tratado comercial del norte T-MEC del gobierno entre 2012 y 2018.

En cuanto a los valles o picos bajos, se encuentran dos: el primero en la época socialista y el segundo en la modernidad. Ambos tienen diferentes extensiones, es decir, su duración varía por estar uno inmerso en un único sexenio gubernamental y el segundo por abarcar más de dos sexenios. Para estos picos de valles o de no inclusión del inglés, resaltan la diferencia ideológica entre ellos, uno de corte socialista y los segundos en el capitalismo de apertura internacional.

Según el análisis genealógico, estas ausencias han ocurrido bajo circunstancias de oposición en el primero y de causalidad en el segundo. Los antecedentes del gobierno socialista aclaran el camino para ubicar una desazón cardenista frente al ímpetu colonialista europeo y norteamericano antes de la mitad de siglo XX y condicionar en lo político y educativo el ideario mexicano, ayudándose de cambios constitucionales. En el segundo periodo de no inclusión del inglés, el capitalismo manifestó una tendencia minimizadora hacia lo educativo y fortalecedora de lo laboral. Además, la inmediatez de requerir un empleo después de terminar la secundaria complicó las inscripciones en las escuelas normales (al haber adquirido el estatus de licenciatura, los estudiantes no podían acceder al término de la secundaria). Así, la obligación de cursar unos años más de preparatoria hizo que las escuelas normales redujeran su tamaño y disminuyeran su alcance, mientras que se acrecentó la migración a los Estados Unidos. De cierta manera, este criterio se tendría en cuenta en unas reformas posteriores al legislar pactos y alianzas multisectoriales, en las que se establecerían conexiones entre formación docente, currículo y competencias para favorecer el crecimiento económico, que conllevarían una tendencia utilitarista sobre la educación desde las políticas educativas.

Estos movimientos ilustran procesos de decisión gubernamental afectados por tensiones sociopolíticas y económicas, que encarnan sentidos de ida y vuelta hacia lo educativo. Eventualmente se repiten por las conexiones entre sí, manifestadas a causa de las contracciones educativas internas en pro de la mejora de las condiciones que no fueron resueltas; en el caso del 
socialismo, fue eliminada de la Constitución al siguiente periodo presidencial, como si hubiera sido un error. Tampoco se solucionaron al convertir las escuelas normales en instituciones de educación superior por el déficit de estudiantes que causó, por la confrontación entre focalizar la teoría o la práctica en el currículo, y aún menos se solucionaron con las alianzas entre gobierno y sindicato que vieron aparecer los deseos de poder de la dirigencia sindical.

Con respecto a la denominación de la asignatura de Inglés durante estos ciclos, discurren cuatro nombres diferentes. En principio fue el nombre de origen «Inglés». Luego fue «Lengua Extranjera» que indicaba su enseñanza a través de la traducción. Recientemente, ha sido designada como «Lengua adicional» en un esfuerzo de implicar su influencia británica. Y en la última reforma de 2017 regresa a ser denominada «Inglés», como en el primer ciclo. Una reiteración en la manera en cómo se nombra entre el ciclo 1 y el ciclo 3 propone sentidos que de nuevo se anclan en la búsqueda de conexiones que mantengan la influencia y el dominio extranjero vigente. Tal fenómeno requiere ser analizado desde horizontes críticos, tanto de los investigadores y maestros como de los futuros docentes normalistas, que permita en conjunto repensar y reformular minuciosamente lo no explícito y lo no lineal a partir de su inserción en la cultura, mas no desde un punto de vista colonialista.

Por último, se vislumbra la emergencia del inglés en las fuerzas que lo han anulado y revitalizado, en las repeticiones de acontecimientos, en errores o fallas que han rodeado a esta asignatura. Este análisis nos ha remitido a evidenciar el deseo de igualdad, las reglas impuestas por culturas o por instancias dominadoras, los elementos detonadores de reformas, las contingencias que han afectado la educación en tanto conflictos internos, extranjeros o de carácter sanitario. A partir de los documentos normativos y de los acontecimientos analizados genealógicamente, se han desglosado circunstancias, figuras y conexiones de robusta significación para la asignatura de Inglés y los sentidos que la transitan y que cobran significado en cada periodo con mayores implicancias laborales futuras para los estudiantes de las escuelas normales mexicanas.

\section{REFERENCIAS}

Aguirre, M. (2010). “Una invención del siglo XIX la escuela primaria (1780-1890)”. En L. Lafarga (Ed.), Diccionario Historia de la Educación en México. Recuperado de https://bit.ly/36I9xUn

Arnaut, A. (1996). Historia de una profesión: Los maestros de educación primaria en México 1887-1994. CIDE.

Baker, C. (2001). Foundations of Bilingual Education and Bilingualism. Recuperado de https://bit. ly/2XbrNT7

Ballín, R. (2007). El Congreso Nacional de Instrucción de 1889, Ponencia presentada en el IX Congreso Nacional de Investigación Educativa, México. Recuperado de https://bit.ly/2B1wZ34

Barrón-Tirado, C. y Pontón-Ramos, C. (2013). "La reforma de la escuela normal de 1997. Algunas consideraciones críticas”. En P. Ducoing (Ed.), La escuela normal: Una mirada desde el otro. Recuperado de https://bit.ly/2XCXJic

Bazant, M. (1993). Historia de la Educación durante el porfiriato. El Colegio de México.

CDPPE (2014) Comité de Direccionamiento de Políticas y Prácticas Educativas. Recomendación CM/ Rec(2014)5. Concejo de Europa. Bruselas 


\section{ESTUDIOS Y ENSAYOS}

Cruz, O. (2007). "Políticas educativas recientes en la formación de profesorado: Algunos elementos conceptuales para su análisis”. En P. Padierna, y R. Mariñez, (Eds.), Educación y comunicación. Tejidos desde el Análisis Político del Discurso (pp. 39-67). Juan Pablos/ Programa de Análisis Político del Discurso e Investigación.

Diario Oficial de la Federación México, (2018). Acuerdo Nº134 de 8 de Junio de 1988, por el que se establece el plan de estudios para la formación de docentes en educación primaria a nivel de licenciatura. Recuperado de https://bit.ly/2X8iKlF

DGESPE (2012). Plan de Estudio 2012. Dirección General de Educación Superior para Profesionales de la Educación. Recuperado de https://bit.ly/3etwOqK

Ducoing, P. (2004). Origen de la Escuela Normal Superior de México. Bogotá: Revista Historia de la Educación Latinoamericana 6(6), 39-56.

Ducoing, P. (2013). La escuela normal: Una mirada desde el otro. Recuperado de https://bit.ly/2XCXJic

Figueroa, M. (2000). La formación de docentes en las escuelas normales: entre las exigencias de la modernidad y las influencias de la tradición. Revista Latinoamericana de Estudios Educativos (México), XXX(1), 117-142. Recuperado de https://bit.ly/3gw6L9m

Foucault, M. (2019). La microfísica del poder. Siglo XXI Editores.

García, J. (2015). La educación lancasteriana en México y América Latina como solución del estado liberal ante el vacío dejado por la iglesia. Boletín Virtual, Julio 4-7, pp. 48-66. Recuperado de https:// bit.ly/2ZVeisz

Hurtado, P. (2002). "Una mirada, una escuela, una profesión: Historia de las escuelas Normales 19211984”. En L.E. Galván (Ed.), Diccionario de historia de la educación en México. Recuperado de https:// bit.ly/36I9xUn

Larios, M., Hernández, G. y Coronado, R. (2016). La labor y logros educativos de Enrique Laubscher en Chihuahua 1889-1890. Ponencia presentada en el XII Congreso Nacional de Investigación Educativa. Educación inicial y básica.

MacLean, K. (2011). Children for Whom English Is an Additional Language. En G. Knowles (Ed.), Supporting Inclusive Practice, 2 ed. (pp.) 26-45. Routledge.

Martínez, R. y Contreras, E. (2016). El papel de la investigación en las escuelas normales. El reto en el perfil de los docentes. Ponencia presentada en el Congreso Nacional las escuelas normales: Una mirada hacia el futuro, Xalapa-Enríquez, Veracruz, pp. 1-12.

Medrano, V., Méndez, E. y Morales, M. (2017). La educación normal en México. Elementos para su análisis. INEE. Recuperado de https://bit.ly/2TLkCig

Mendoza, M. (2017). Breve resumen histórico de la enseñanza del inglés en los planes de estudio de educación básica y media superior. Pobacma 6(1), pp.55-60.

Meneses-Morales, E. (1973). Tendencias educativas oficiales en México 1821-1911. La Problemática de la Educación Mexicana en el siglo XIX y principios del siglo XX. Editorial Trillas.

Meneses-Morales, E. (1991). Tendencias Educativas Oficiales en México 1964-1976. CEE-UIA.

Meneses-Morales, E. (1998c). Tendencias educativas oficiales en México 1934-1964. CEE-UIA

Meyer, J. (2011). Dos siglos, dos naciones México y Francia 1810 - 2010. CIDE 72. Recuperado de https:// bit.ly/3d51KlU 


\section{ESTUDIOS Y ENSAYOS}

Meyer, L. (2015). Felipe Calderón o el infortunio de una transición. Foro internacional, 55(1), 16-44. Recuperado de https://bit.ly/3ekFcOe

Mora, J. (1979). Los maestros y la práctica de la educación socialista. Universidad Pedagógica Nacional 29, (1) Recuperado de https://bit.ly/2XEH8dA

Muñoz, M. (2015). La enseñanza del inglés en planes y programas de estudio en las escuelas normales de México. Praxis Investigativa ReDIE 7(12), pp. 85-95. Recuperado de https://bit.ly/2M5T9U9

Navarrete, Z. (2015). Formación de profesores en las escuelas normales de México siglo XX. Revista Historia de la Educación Latinoamericana 17(25), 17-34. Disponible en https://bit.ly/2ZHFSsT

Ortega, R. y Somuano, M. (2015). El periodo presidencial de Felipe Calderón Hinojosa. Foro internacional, 55(1), 5-15. Recuperado de https://bit.ly/3gsY2og

Quiñones, L. (2001). La Benemérita y Centenaria Escuela Normal del Estado de Durango. CXXX Aniversario. México, Benemérita y Centenaria Escuela Normal del Estado de Durango. Recuperado de https:// bit.ly/2ZKJk6i

Ramos, C. (1977). Enrique Rébsamen ideólogo educativo. En Primer anuario Centro de Estudios Históricos. Recuperado de https://bit.ly/3erGyqq

Rodríguez y Romero, D. (2018). Compilación para la sesión de la Sociedad de Estudios Históricos del Metodismo en México. Recuperado de https://bit.ly/3eokU6z

Rodríguez, L. (2006). Políticas educativas 1940-1970: Una reflexión desde la docencia. Tesis de Licenciatura). Universidad Pedagógica Nacional Unidad D.F. Centro. México, Recuperado de https:// bit.ly/2XyTLXK

Salazar, M., Chapa, M. y Cantillo, M. (2017). La enseñanza del inglés en la escuela normal Pablo Livas: De lo extracurricular a la certificación. Ponencia presentada en el Congreso Mexicano Investigación Educativa. Mérida, Yucatán. pp.1-8.

SEGUNDO CONGRESO NACIONAL DE INSTRUCCIÓN (1981). Informes y Resoluciones. Imprenta de Francisco Díaz de León. México. Universidad de Nuevo León. Recuperado de https://bit.ly/2AehPaw

SEP (2002). Plan de estudios 1997 Programa para la Transformación y Fortalecimiento Académicos de las Escuelas Normales. Secretaría de Educación Pública, México: SEP. Recuperado de https://bit. ly/2yDdugr

SEP (2017). Estrategia de fortalecimiento y transformación de las escuelas normales. Secretaría de Educación Pública, Recuperado de https://bit.ly/2XAMQgI

Staples, A. (1992). “Alfabeto y catecismo, salvación del nuevo país”, en A. Hernández y M. Miño (Eds.), Lecturas de Historia Mexicana (pp.69-92). El Colegio de México.

Staples, A. (2005). Recuento de una batalla inconclusa. La educación mexicana de Iturbide a Juárez. El Colegio de México.

Tanck, D. (1977). “La escuela ilustrada -1786 -1836- Educación primaria en la ciudad de México, México”, pp.140-142). Colegio de México, Nueva Serie 22.

Tanck, D. (1992). “Las escuelas lancasterianas en la Ciudad de México:1822-1842”, en A. Hernández y M. Miño (Eds.), Lecturas de historia mexicana (pp.49-68). El Colegio de México. 


\section{ESTUDIOS Y ENSAYOS}

Vera, K. (2006). Aportes de Don Enrique C. Rébsamen a la educación mexicana. (Tesis de Licenciatura). Universidad Pedagógica Nacional Unidad Ajusco. Recuperado de https://bit.ly/3cb38Cm

Womack, Jr. J. (2012). La economía de México durante la Revolución, 1910-1920: historiografía y análisis. Argumentos (México, D.F.), 25(69), 13-56. Recuperado de https://bit.ly/3ekJnJU

Yurén, M. (2009). Reformas Curriculares en la Formación de Docentes en México. Educação\& Realidade, 34 (1), 33-48. Recuperado de https://bit.ly/2Af5l2e 